\title{
Phenotypic and Functional Properties of Porcine Dedifferentiated Fat Cells during the Long-Term Culture In Vitro
}

\author{
Xuewu Peng, ${ }^{1}$ Tongxing Song, ${ }^{1}$ Xiaoming Hu, ${ }^{1}$ Yuanfei Zhou, ${ }^{1,2}$ Hongkui Wei, ${ }^{1,2}$ \\ Jian Peng, ${ }^{1,2}$ and Siwen Jiang ${ }^{2,3}$ \\ ${ }^{1}$ Department of Animal Nutrition and Feed Science, College of Animal Science and Technology, Huazhong Agriculture University, \\ Wuhan 430070, China \\ ${ }^{2}$ The Cooperative Innovation Center for Sustainable Pig Production, Wuhan 430070, China \\ ${ }^{3}$ Key Laboratory of Swine Genetics and Breeding of Agricultural Ministry and Key Laboratory of Agricultural Animal Genetics, \\ Breeding and Reproduction of Ministry of Education, College of Animal Science and Technology, Huazhong Agriculture University, \\ Wuhan 430070, China
}

Correspondence should be addressed to Jian Peng; pengjian@mail.hzau.edu.cn and Siwen Jiang; jiangsiwen@mail.hzau.edu.cn Received 20 January 2015; Revised 25 April 2015; Accepted 30 April 2015

Academic Editor: Matthew B. Wheeler

Copyright (C) 2015 Xuewu Peng et al. This is an open access article distributed under the Creative Commons Attribution License, which permits unrestricted use, distribution, and reproduction in any medium, provided the original work is properly cited.

\begin{abstract}
It has been proved that terminally differentiated mature adipocytes possess abilities to dedifferentiate into fibroblast-like progeny cells with self-renewal and multiple differentiation, termed dedifferentiated fat (DFAT) cells. However, the biological properties of DFAT cells during long-term culture in vitro have not been elucidated. Here, we obtained fibroblast-like morphology of porcine DFAT cells by ceiling culture. During the dedifferentiation process, round mature adipocytes with single large lipid droplets changed into spindle-shaped cells accompanied by the adipogenic markers PPAR $, a P 2, L P L$, and Adiponectin significant downregulation. Flow cytometric analysis showed that porcine DFAT cells displayed similar cell-surface antigen profile to mesenchymal stem cells (MSCs). Furthermore, different passages of porcine DFAT cells during long-term culture in vitro retained high levels of cell viabilities $(>97 \%)$, efficient proliferative capacity including population doubling time ranged from $20 \mathrm{~h}$ to $22 \mathrm{~h}$ and population doubling reached $47.40 \pm 1.64$ by 58 days of culture. In addition, porcine DFAT cells maintained the multiple differentiation capabilities into adipocytes, osteoblasts, and skeletal myocytes and displayed normal chromosomal karyotypes for prolonged passaging. Therefore, porcine DFAT cells may be a novel model of stem cells for studying the functions of gene in the different biological events.
\end{abstract}

\section{Introduction}

Mesenchymal stem cells (MSCs) are multipotent cells derived from the stromal fraction of many adult tissues including bone marrow [1], adipose tissue [2], skeletal muscle [3], peripheral blood [4], and umbilical cord [5]. MSCs possess the capacity of self-renewal and multipotency to differentiate into adipocytes, osteoblasts, and chondrocytes [6]. Compared with bone marrow derived mesenchymal stem cells (BMSCs), adipose-derived stem cells (ASCs) isolated from adipose tissue have been an attractive source of MSCs due to the easy accessibility with little donor site injury [7]. However, the heterogeneity and insufficient cell numbers of ASCs limit the clinical applications.

Mature adipocytes constitute more than $90 \%$ of adipose tissue volume and are the most abundant cell type in adipose tissue [8]. Recent studies have reported that terminally differentiated mature adipocytes possess ability to undergo dedifferentiation and form proliferative, multipotent, and fibroblast-like progeny cells by ceiling culture, named dedifferentiated fat (DFAT) cells $[9,10]$. DFAT cells exhibit similar properties to BMSCs and may be a novel adult stem cell source for tissue engineering and cell therapy [11] because of the more abundant cell numbers and the high purity as 
compared to ASCs [12]. However, the functional characteristics of DFAT cells during long-term culture in vitro remain elusive. Pigs are generally considered as large animal model which are similar to human in genetic and physiological characteristics and could be an ideal model for biomedicine.

In the present study, we attempt to harvest porcine DFAT cells by ceiling culture of mature adipocytes isolated from adipose tissue. Then, we identify biological characteristics of porcine DFAT cells including morphology, viability, immunophenotype, differentiation potential, and chromosomal karyotypes during the long-term culture in vitro, aiming to further define the properties of porcine DFAT cells by prolonged passaging.

\section{Materials and Methods}

2.1. Isolation, Purification, and Culture of Porcine Mature Adipocytes. All animal experiments were approved by the Animal Care and Use Committee of Huazhong Agricultural University, China. Mature adipocytes were isolated from five-day-old male landrace piglet with a slight modification described previously [9]. Briefly, subcutaneous adipose tissue was obtained in sterile condition and washed with phosphatebuffered saline (PBS). Then, the adipose tissue was minced into small pieces and digested in $0.1 \%(\mathrm{w} / \mathrm{v})$ collagenase type I (Sigma, USA) at $37^{\circ} \mathrm{C}$ for $1 \mathrm{~h}$ with gentle agitation. After filtration and centrifugation at $300 \mathrm{~g}$ for $10 \mathrm{~min}$, the floating mature adipocytes in the top layer were collected. After the wash with Dulbecco's modified Eagle's medium (DMEM, Invitrogen, USA) for three times, cells $\left(5 \times 10^{4}\right)$ were plated in $12.5 \mathrm{~cm}^{2}$ culture flasks (Jet, Canada) completely filled with DMEM supplemented with $20 \%$ fetal bovine serum (FBS, Invitrogen, USA) and were incubated at $37^{\circ} \mathrm{C}$ in $5 \% \mathrm{CO}_{2}$. Culture flasks containing mature adipocytes were inverted and the floating adipocytes attached to the top inner ceiling surface.

To obtain highly purified mature adipocytes, cells were purified with differential plating until there were no other cells to eliminate contamination [13]. After 7 days of ceiling culture, the culture flasks were inverted so that the cells were on the bottom and the medium was changed every 3 days until the cells reached confluence.

Primary DFAT cells were digested with $0.25 \%$ (w/v) trypsin-EDTA (Invitrogen) and long-term cultured in growth medium of low-glucose DMEM supplemented with 15\% FBS, $10 \mathrm{ng} / \mathrm{mL}$ basic fibroblast growth factor (bFGF) (Peprotech, USA), and $1 \%$ penicillin/streptomycin (Invitrogen, USA).

2.2. Flow Cytometric Analysis. Porcine DFAT cells at passage 4 (P4) were detected by flow cytometric for cell surface antigen. The cells were digested with $0.25 \%$ trypsin-EDTA and incubated with primary antibody CD29 (552369, BD, USA), CD31 (MCA1746PET, AbD Serotec, USA), CD34 (GB12013, China), CD44 (ab19622, Abcam, USA), and CD90 (562245, $\mathrm{BD}, \mathrm{USA}$ ) for $30 \mathrm{~min}$ at $4^{\circ} \mathrm{C}$ and further they were incubated for $30 \mathrm{~min}$ at $4^{\circ} \mathrm{C}$ with FITC or CY3 conjugated secondary antibody. Cell fluorescence was detected by FACSCalibur instrument (Becton Dickinson, USA). The data were analyzed using CellQuest Software (Becton Dickinson, USA).

2.3. Cell Viability Assay. The cell viability was assessed by trypan blue staining. The stain of the porcine DFAT cells was performed according to the manufacturer's instructions of Countess Automated (Invitrogen, USA).

2.4. Growth Kinetics Analysis. Growth kinetics of porcine DFAT cells were analyzed by detection of cumulative population doubling (CPD) and population doubling time (PDT). Porcine DFAT cells at P1 were plated at $1 \times 10^{4} / \mathrm{cm}^{2}$ in growth medium until $70 \%-80 \%$ confluence and the cells were digested and replated at $1 \times 10^{4} / \mathrm{cm}^{2}$ in growth medium. The calculation of population doubling was performed as the formula: PDs $=\log _{2}$ (number of harvested cells/number of seeded cells) [11]. Cumulative population doubling (CPD) was calculated by the total numbers of PDs in the previous passages. PDT was calculated by the formula: $\mathrm{PDT}=t / \mathrm{PD}$, where $t$ is the culture time during this passage [14].

2.5. RNA Extraction, Reverse Transcription, and Real-Time $P C R$. Total RNA was extracted using TRIzol reagent (Invitrogen, USA) according to the manufacturer's instructions. Total RNA ( $1 \mu \mathrm{g})$ was performed by reverse transcription (RT) using reverse transcription kit (Toyobo, Japan). Realtime PCR was performed with iTaq Universal SYBR Green Supermix (Bio-Rad, USA) using the CFX96 Detection System (Bio-Rad, USA). The relative expression levels of PPAR $\gamma, a P 2, L P L$, Adiponectin, and Runx2 mRNA were normalized to $\beta$-actin. The primers are as follows: $\beta$ actin forward: $5^{\prime}$-CCAGGTCATCACCATCGG-3', reverse: $5^{\prime}$-CCGTGTTGGCGTAGAGGT-3'; PPAR $\gamma$ forward: $5^{\prime}$ AGAGTATGCCAAGAACATCC-3', reverse: $5^{\prime}$-AGGTCGCTGTCATCTAATTC-3 $3^{\prime}$; aP2 forward: $5^{\prime}$-AAGTCAAGAGCACCATAACC- $3^{\prime}$, reverse: $5^{\prime}$-GATACATTCCACCACCAACT- $3^{\prime}$; LPL forward: $5^{\prime}$-CGACTCTCTGTTGAATGAAG-3', reverse: $5^{\prime}$-TTGGCTCTGACCTTATTGATC-3'; Adiponectin forward: $5^{\prime}$-TTGAAGGATGTGAAGGTCAG$3^{\prime}$, reverse: $5^{\prime}$-CAATGTTGTGGTAGAGAAGG-3'; Runx2 forward: $5^{\prime}$-CAGACCAGCAGCACTCCATA-3', reverse: $5^{\prime}$-AACGCCATCGTTCTGGTTAG-3'; MyoG forward: $5^{\prime}$ CCAACCAGCGGCTGCCTAAAG-3', reverse: $5^{\prime}$-ATTGTGGGCGTCTGTAGGGTCA-3'.

2.6. Adipogenic, Osteogenic, and Myogenic Differentiation. For adipogenic differentiation, DFAT cells at $80 \%-90 \%$ confluence were treated with high-glucose DMEM containing $10 \% \mathrm{FBS}, 1 \mu \mathrm{M}$ dexamethasone, $500 \mu \mathrm{M}$ isobutylmethylxanthine (IBMX), $10 \mu \mathrm{g} / \mathrm{mL}$ insulin, and $200 \mu \mathrm{M}$ indomethacin (all reagents were from Sigma, USA) for 12 days. The lipid droplets were confirmed by Oil Red $\mathrm{O}$ stain and extracted total RNA for RT-PCR analysis.

For osteogenic differentiation, DFAT cells at 50\%-60\% confluence were induced with high-glucose DMEM containing $10 \%$ FBS, $0.1 \mu \mathrm{M}$ dexamethasone, $10 \mathrm{mM} \beta$-glycerophosphate, and $50 \mathrm{mM}$ ascorbic acid (all reagents were from Sigma, USA) for 21 days and induction medium was 
replaced every 3 days. The mineralized deposits were visualized by Alizarin Red stain and extracted total RNA for RTPCR analysis.

For myogenic differentiation, DFAT cells at 70\%-80\% confluence were induced with low-glucose DMEM containing $200 \mathrm{ng} / \mathrm{mL}$ Galectin-1 (Prospec, USA) protein for 21 days, and medium was changed every 3 days. Myogenic markers Desmin and $\mathrm{MyHC}$ were assessed by immunofluorescence staining.

2.7. Immunofluorescence Analysis. Myogenic differentiation of DFAT cells was detected by immunofluorescence staining. The induced cells were fixed with $4 \%$ paraformaldehyde for $10 \mathrm{~min}$ and treated with $0.1 \%$ Triton X-100. Then, cells were blocked with $5 \%$ bovine serum albumin (BSA, Sigma, USA) and incubated with primary antibodies Desmin (1:100, sc-14026, Santa Cruz, USA), and MyHC (1:100, ab11083, Abcam, USA) overnight at $4^{\circ} \mathrm{C}$. Thereafter, TRITC and FITC conjugated secondary antibodies were incubated at room temperature for $1 \mathrm{~h}$. The cell nucleus was stained with DAPI.

2.8. Karyotype Analysis. Chromosomal karyotyping of early, middle, and late passages of DFAT cells was performed as previously reported [15]. At least 20 metaphases were acquired by Leica microscope.

2.9. Statistical Analysis. The results are presented as means \pm standard deviations. All experiments were repeated a minimum of three times. The statistical significance was assessed by one way analysis of variance (ANOVA) along with Duncan multiple range test. $p$ values $<0.05$ were considered significant.

\section{Results}

3.1. Morphological and Gene Expression Changes during the Ceiling Culture of Porcine Mature Adipocytes. Porcine mature adipocytes were isolated and purified with collagenase digestion and differential plating method. During the ceiling culture, the mature adipocytes containing single large lipid droplets (Figure 1(a)-A) attached to the top surface of the culture flasks in 1-2 days and changed morphology in following about 2 weeks (Figure 1(a)-A-F). After mature adipocytes firmly attached to the flasks, the shape became to elongated and spread "tentacles" at day 3. Then, the single large lipid droplets in the cytoplasm gradually were released and extruded out the cells on days 6-14, leading to the following: the cell changed into fibroblast-like morphology and did not contain lipid droplets (Figure 1(a)-F). The culture flasks were inverted, the medium was changed at days 710 , and the cells proliferated and reached confluence. After trypsinization, P1 DFAT cells uniformly exhibited fibroblastlike morphology and we refer to these cells as DFAT cells in this paper (Figure 1(b)).

Next, we detected the gene expression changes of dedifferentiation of mature adipocytes (MAs) during ceiling culture by using real-time RT-PCR. Adipogenic related genes including PPAR $\gamma, a P 2, L P L$, and Adiponectin were highly expressed in mature adipocytes, whereas these genes were almost not expressed in DFAT cells (Figure 1(c)). These results suggest that DFAT cells lose the functional characteristics of mature adipocytes and are consistent with previous reports $[9,11]$.

3.2. Cell-Surface Antigen Expression Profile of DFAT Cells. The cell-surface antigen of porcine DFAT cells at P4 was examined by flow cytometric analysis. DFAT cells were positive for mesenchymal surface antigen including CD44 (91.51\%), CD29 (71.61\%), and CD90 (86.84\%), which are typically expressed in MSCs. The expression levels of endothelial cells marker CD31 (1.26\%) and hematopoietic cells marker CD34 (3.59\%) were low in porcine DFAT cells (Figure 2). This expression profile was similar to BMSCs and consistent with that previously reported $[9,11]$.

3.3. Cell Morphology and Viability of Porcine DFAT Cells during Long-Term Culture. DFAT cells were routinely cultured for up to 60 passages in vitro and maintained typical spindle-shaped morphology (Figure 3(a)). DFAT cells were fibroblast-like in shape and the cytoplasm was homologous without vacuoles and smudges and displayed a good growth status during the long-term culture.

To examine the cell activity during prolonged culture, cell viability of early (P1-P10), middle (P20-P30), and late (P50$\mathrm{P} 60)$ passages was examined by trypan blue staining. Different passages of DFAT cells all remained high viability (>97\%) and showed no significance in each other $(98.00 \% \pm 0.82 \%$ in $\mathrm{P} 5,98.60 \% \pm 1.14 \%$ in $\mathrm{P} 29$, and $99.25 \% \pm 0.96 \%$ in $\mathrm{P} 59)$ $(p>0.05)$ (Figure 3(b)), suggesting that DFAT cells maintain high and stable activity during long-term culture in vitro.

3.4. Growth Kinetics of DFAT Cells during Prolonged Culture In Vitro. The population doubling time (PDT) of early passage (P10) was $21.68 \pm 1.12 \mathrm{~h}$, which was similar to those of middle (P30) $(20.72 \pm 1.69 \mathrm{~h})$ and late $(\mathrm{P} 60)$ passages $(21.46 \pm 0.63 \mathrm{~h})$ $(p>0.05)$ (Figure 3(c)). Moreover, the cumulative population doubling (CPD) was $47.40 \pm 1.64$ in 58 days during 16 passages of culture and showed a linear correlation with passage number, indicating a relatively constant population doubling rate over the range studied ( $y=3.0442 x-0.4881$, where $y$ was the CPD, $x$ was passage, and $R^{2}=0.9987$ ) (Figure $3(\mathrm{~d})$ ). The results suggest that porcine DFAT cells retain stable growth characteristic and efficient proliferative potential.

3.5. Chromosomal Karyotyping Analysis. Chromosomal karyotypes of P8, P30, and P60 DFAT cells were analysed by Giemsa staining. Different passages of porcine DFAT cells all retained a normal diploid karyotype (38 chromosomes) (Figure 3(e)) after long-term culture indicated the stability of genetic characteristic.

3.6. Multilineage Differentiation of Porcine DFAT Cells. Adipogenic differentiation assessment of P3, P20, and P50 DFAT cells was induced for 12 days. Oil Red $\mathrm{O}$ staining represented all passages of DFAT cells accumulated large numbers of lipids and accompanied with significant upregulation of adipogenic differentiation marker $P P A R \gamma$ expression after 


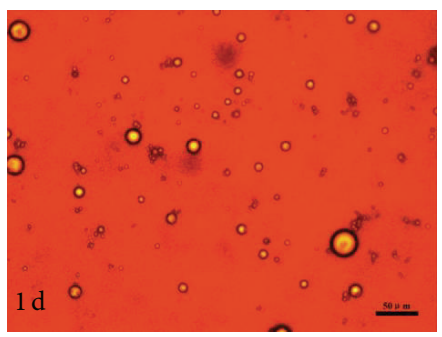

(A)

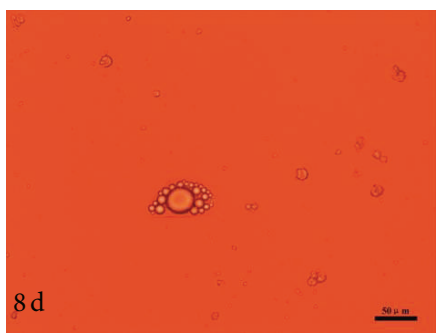

(D)

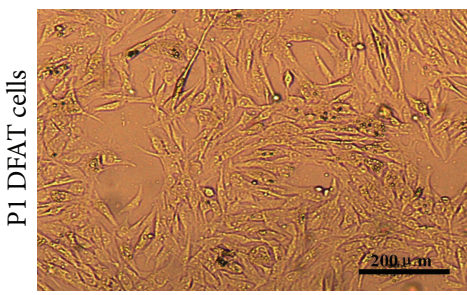

(b)

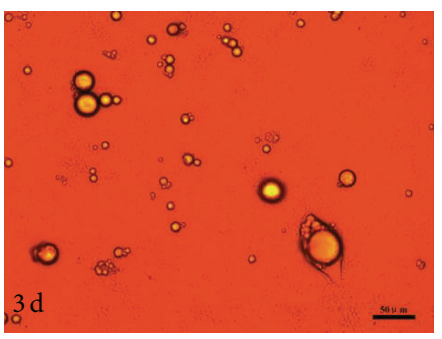

(B)

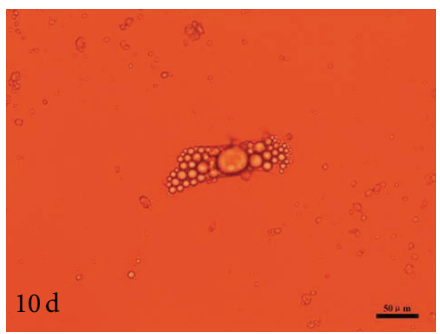

(E)

(a)

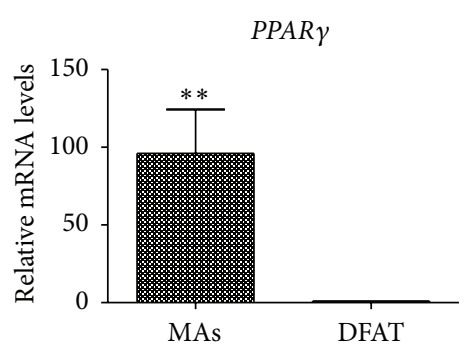

LPL

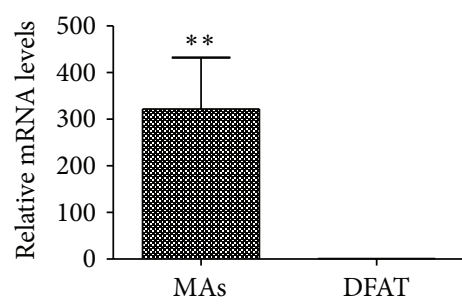

(c)

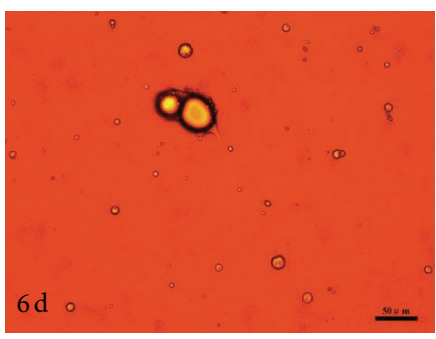

(C)

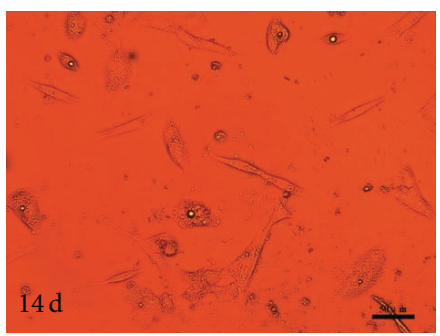

(F)
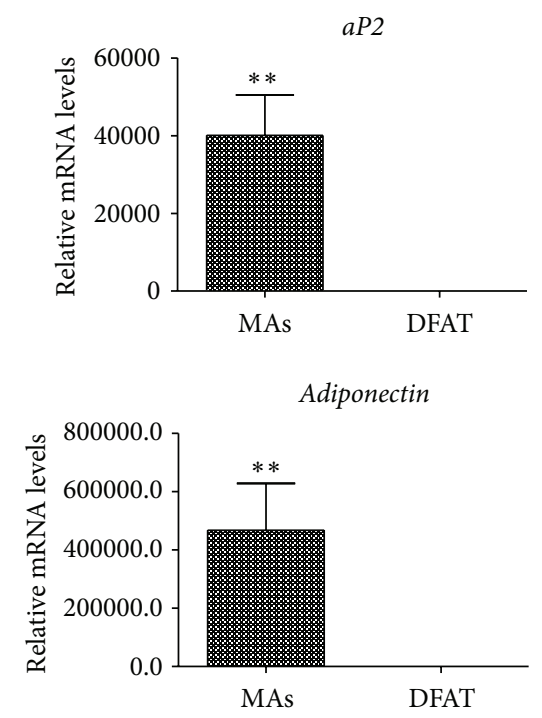

FIgURE 1: Morphological and gene expression changes during the dedifferentiation from porcine mature adipocytes to DFAT cells by ceiling culture. (a) Morphological changes of the dedifferentiation process. During the ceiling culture, mature adipocytes contained large single lipid droplets attached to the flasks firmly (A) at days 1-2 and the cells became oval shape at day 3 (B). Then, the lipid droplets were released and extruded out the cells at days 6-10 (C-E). Finally, the cells changed into fibroblast-like DFAT cells (F), bar, $50 \mu \mathrm{m}$. (b) Primary DFAT cells at P1 after trypsinization, bar, $200 \mu \mathrm{m}$. (c) The gene expression of adipogenic markers between mature adipocytes (MAs) and DFAT cells. The mRNA relative levels of qPCR PPAR $\gamma, a P 2, L P L$, and Adiponectin were detected by qPCR. Values are the mean \pm SD of triplicate dishes. ${ }^{* *} p<0.01$.

induction. Furthermore, the expression of PPAR $\gamma$ showed no differences when inducing adipogenic differentiation for 12 days among the DFAT cells of passages 3, 20, and 50 (Figure 4(a)).

Osteogenic differentiation was performed for P3, P20, and P50 DFAT cells in osteogenic induction medium for 21 days. Mineralized matrix aggregates were observed in all passages of DFAT cells by staining for alizarin red. Furthermore, the expression of osteogenic differentiation marker
Runx2 was significantly increased after induction, and the level of Runx2 revealed no changes when inducing osteogenic differentiation for 21 days among the DFAT cells of passages 3, 20, and 50 (Figure 4(b)).

Myogenic differentiation of P15 and P25 DFAT cells by Galectin-1 protein for 21 days was induced. Myogenic differentiation was confirmed by the positive staining of Desmin and $\mathrm{MyHC}$, the early and late markers of myogenic differentiation, respectively. Multinucleated cells were observed and 

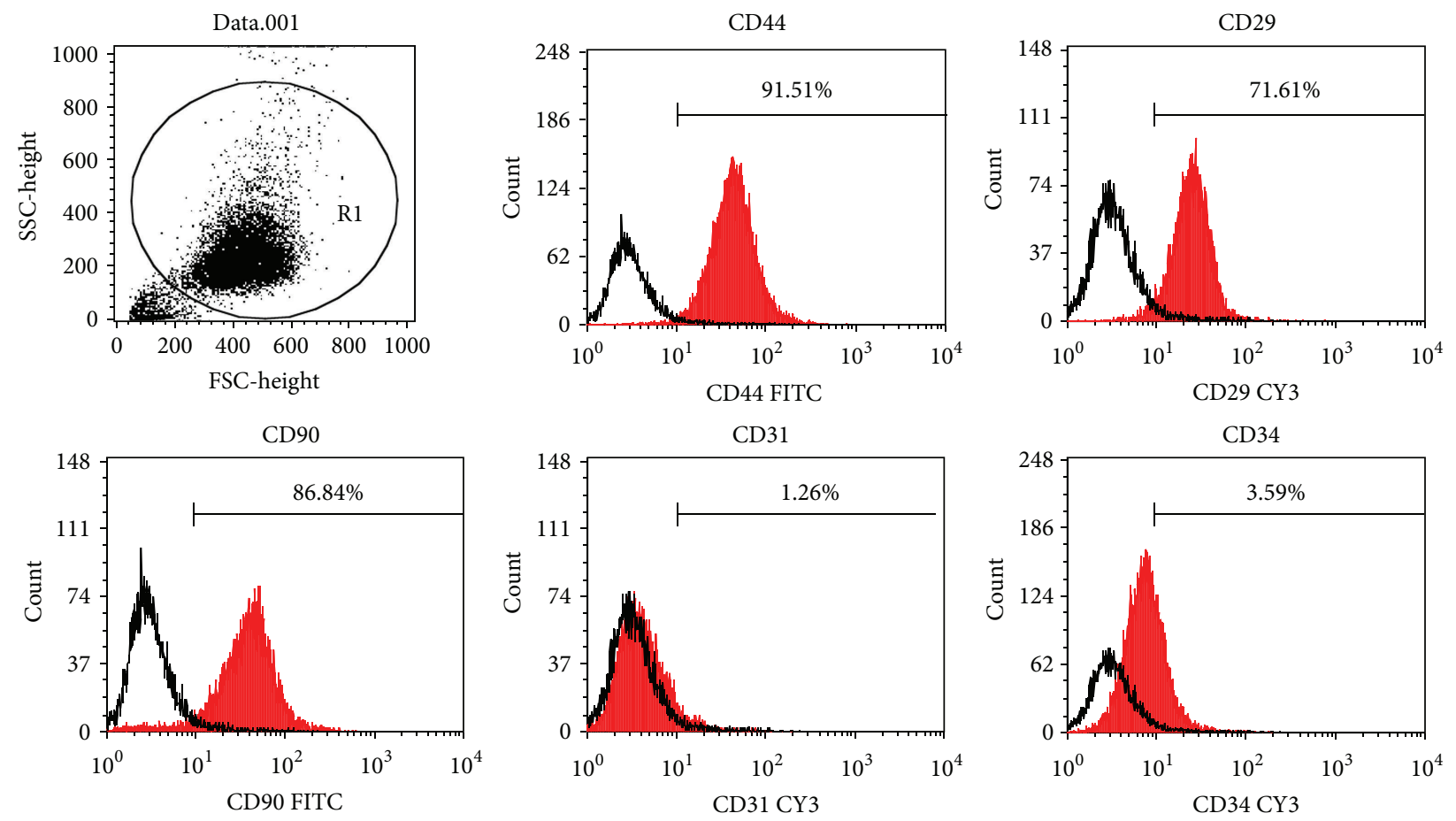

Figure 2: Expression of cell surface antigens on porcine DFAT cells. Expressions of CD44, CD29, CD90, CD31, and CD34 were detected by FITC or CY3 conjugated antibody of P4 DFAT cells.

this indicated that DFAT cells could be induced into skeletal muscle cells. Real-time PCR data analysis found that the myogenic differentiation marker $M y o G$ mRNA was higher after induction 21 day when compared to 0 day. Furthermore, the differentiation ability was changeless among the DFAT cells of passages 3, 20, and 50 (Figure 4(c)).

\section{Discussion}

Adipose tissue has been considered not only an extremely important organ for energy metabolism, but also an endocrine organ that secretes hormones including leptin and adiponectin $[16,17]$. Moreover, adipose tissue can be an excellent source for adult stem cells. The DFAT cells, derived from mature adipocytes by ceiling culture in vitro, would be a more attractive choice than ASCs [18].

Ceiling culture is commonly used to establish DFAT cells and harvesting high purity of mature adipocytes is crucial [19]. The purity of mature adipocytes could reach $98 \%$ confirmed by fluorescence activated cell sorting (FACS) [20]. In this study, we isolated porcine mature adipocytes by collagenase digestion. To eliminate the contaminant cells including preadipocytes and ASCs, multiple cycles of centrifugation and filtration are critical to purify mature adipocytes. Furthermore, differential plating method is effective to obtain mature adipocytes with large single lipid droplet (Figure 1(a)A). Adipocytes dedifferentiation could be induced by a canonical Wnt ligand, causing the morphology to change into fibroblast-like shape and expression of adipogenic markers to downregulate [21]. Our results turn out that porcine mature adipocytes undergo dedifferentiation during ceiling culture with a series of morphological changes (Figure 1(a)A-F), causing fibroblast-like porcine DFAT cells to appear. Gene expression changes between mature adipocytes and DFAT cells indicate that DFAT cells lose the functional characteristics of adipocytes, which is consistent with that previously reported $[9,22]$.

Although DFAT cells are easily established by ceiling culture in many studies and DMEM medium containing 20\% FBS was usually used for passaging culture of DFAT cells [9, $11,12]$, the optimal culture system for DFAT cells during the long-term subculture in vitro has not been established so far. However, high concentration of FBS containing proliferative inhibitors and differentiation promoters may affect the characteristics during the long-term subculture, which resulting in inhibition of cell proliferation, untimely differentiation, and even malignant transformation of MSCs [23, 24]. Basic fibroblast growth factor was recognized as a potent growth factor for cell proliferation and maintained undifferentiated status, which was suitable for cell long-term subculture in vitro [25]. In this study, culture mediums of DMEM medium containing $15 \%$ FBS and $10 \mathrm{ng} / \mathrm{mL}$ bFGF are used for longterm culture of porcine DFAT cells. In this culture condition, porcine DFAT cells can be propagated for 60 passages and still maintain the normal spindle-shaped morphology with high efficient cell viability ( $>97 \%)$.

DFAT cells possess high capacity of proliferation in vitro [26]. Growth kinetics of porcine DFAT cells including CPD and PDT usually evaluated the proliferative capacity. The PDT at all passages of DFAT cells is less than $24 \mathrm{~h}$ and is 

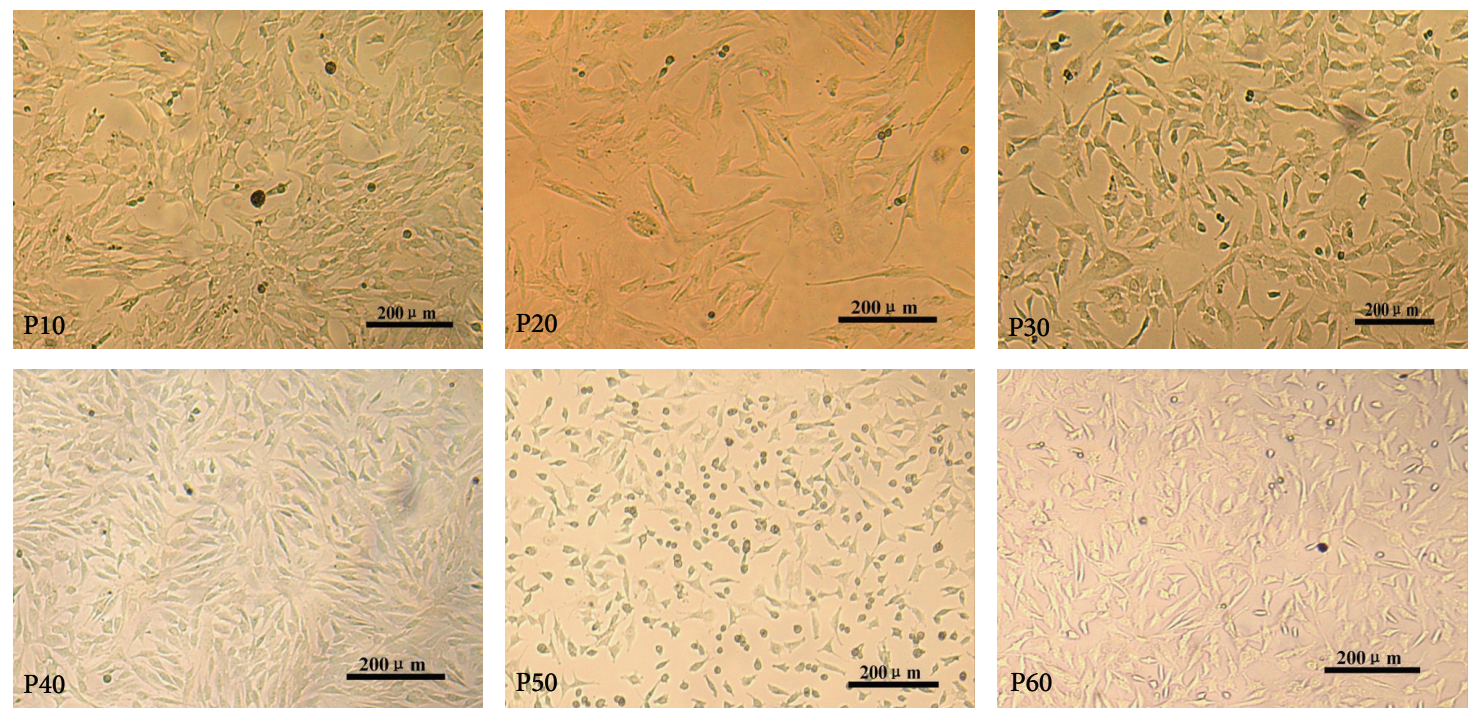

(a)

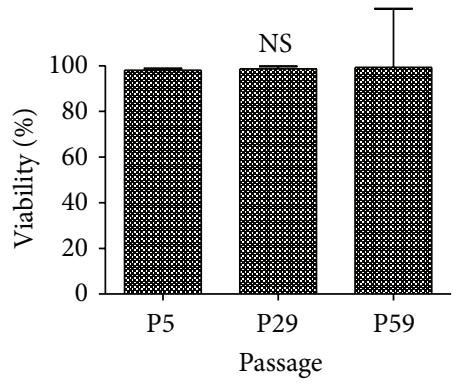

(b)

P8

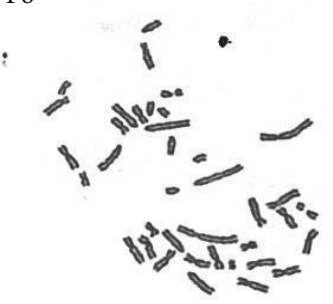

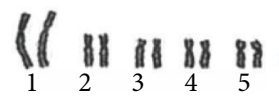

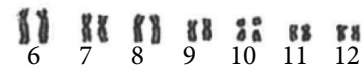

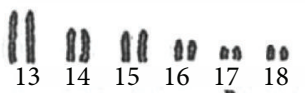

$\begin{array}{lll}8 & \\ X & Y\end{array}$

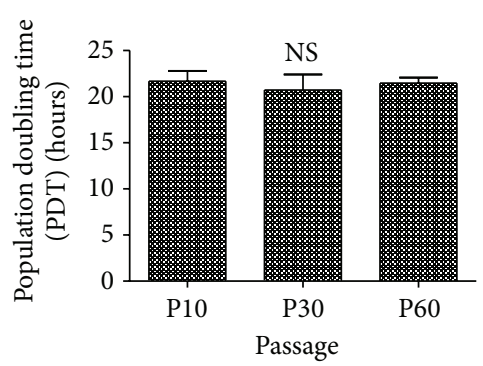

(c)

P30

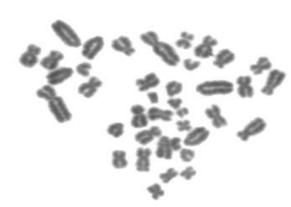

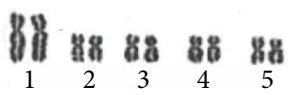

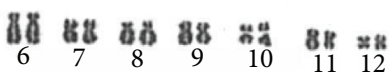

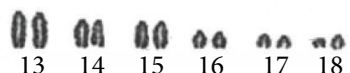

$\stackrel{\text { : }}{X} \stackrel{\text { : }}{ }$

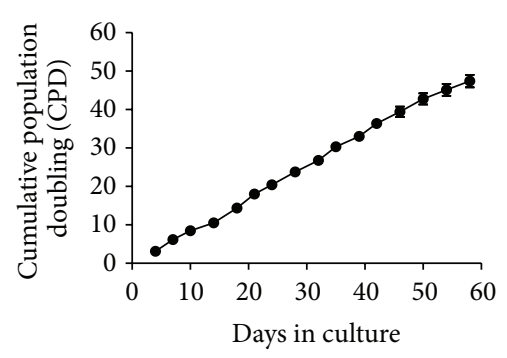

(d)

P60

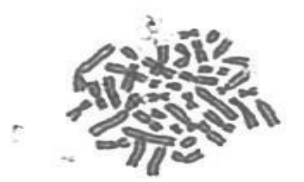

3i) की मी पा तो

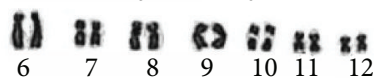

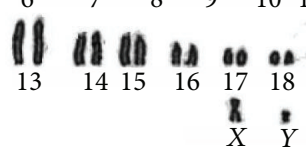

(e)

FIGURE 3: Characterization of porcine DFAT cells during the long-term culture. (a) Different passages of DFAT cells at P10, P20, P30, P40, P50, and P60 uniformly displayed fibroblast-like morphology, bar, $50 \mu \mathrm{m}$. (b) Quantification of viability of P5, P29, and P59 DFAT cells by trypan blue staining. (c) Population doubling time (PDT) of DFAT cells at P10, P30, and P60 was 21.68 $\pm 1.12 \mathrm{~h}, 20.72 \pm 1.69 \mathrm{~h}$, and $21.46 \pm 0.63 \mathrm{~h}$, respectively. (d) Cumulative population doubling (CPD) detected from P1 to P16 reached $47.40 \pm 1.64$ in 58-day culture. (e) The karyotype analysis of P8, P30, and P60 DFAT cells with Giemsa staining. Values are the mean \pm SD of triplicate dishes. NS means no significant difference $(p \geq 0.05)$. 

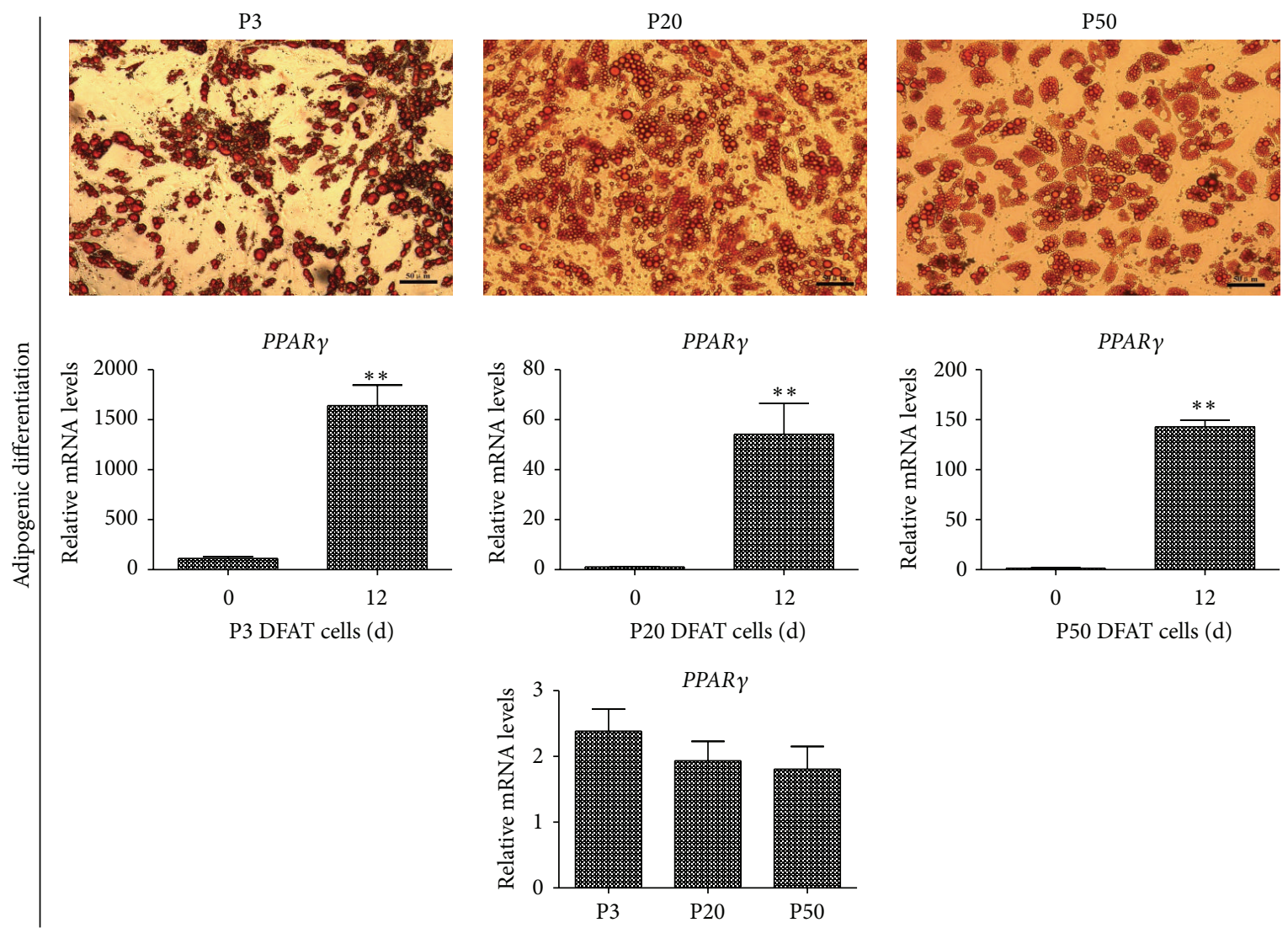

(a)
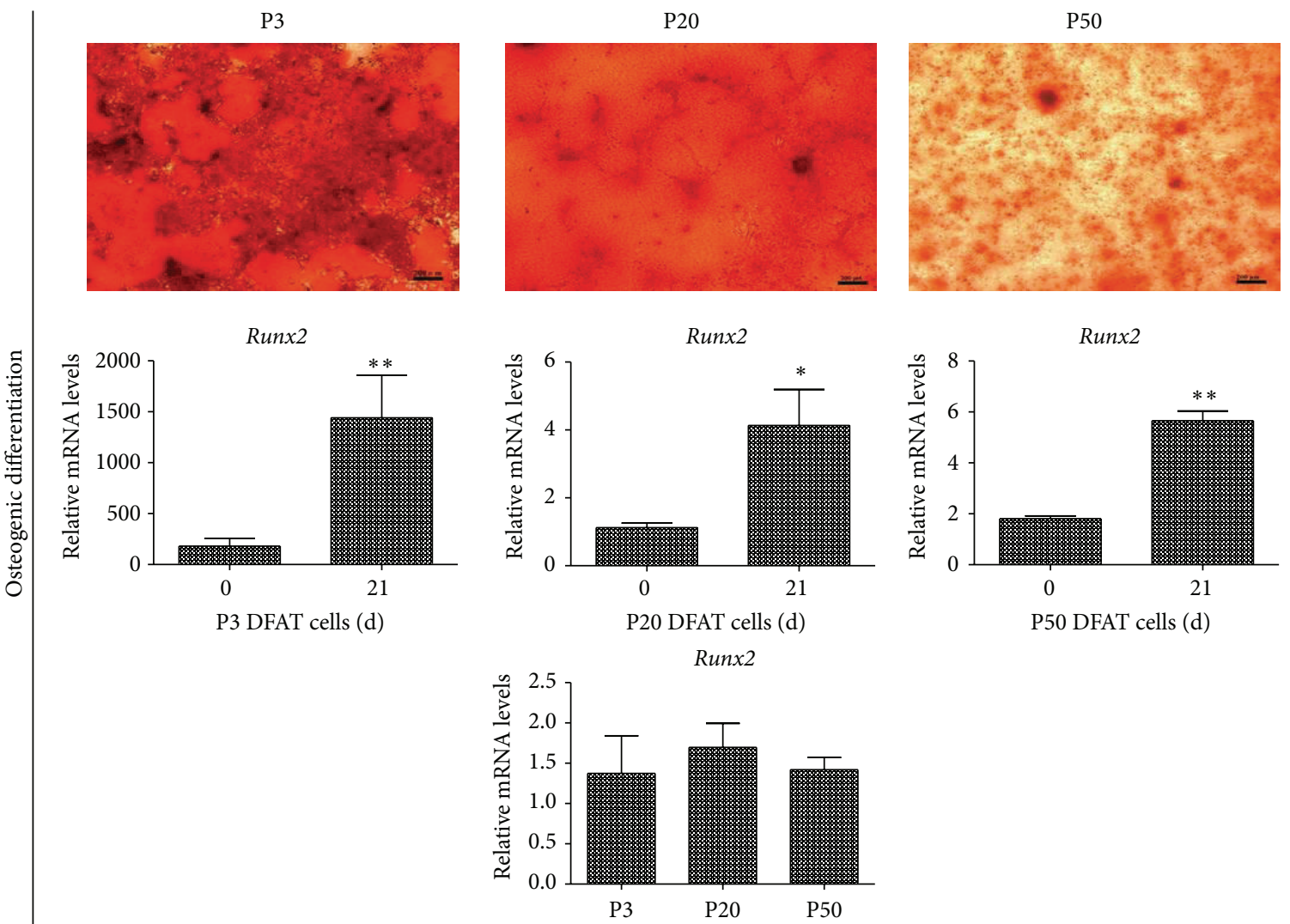

(b)

FIgUre 4: Continued. 


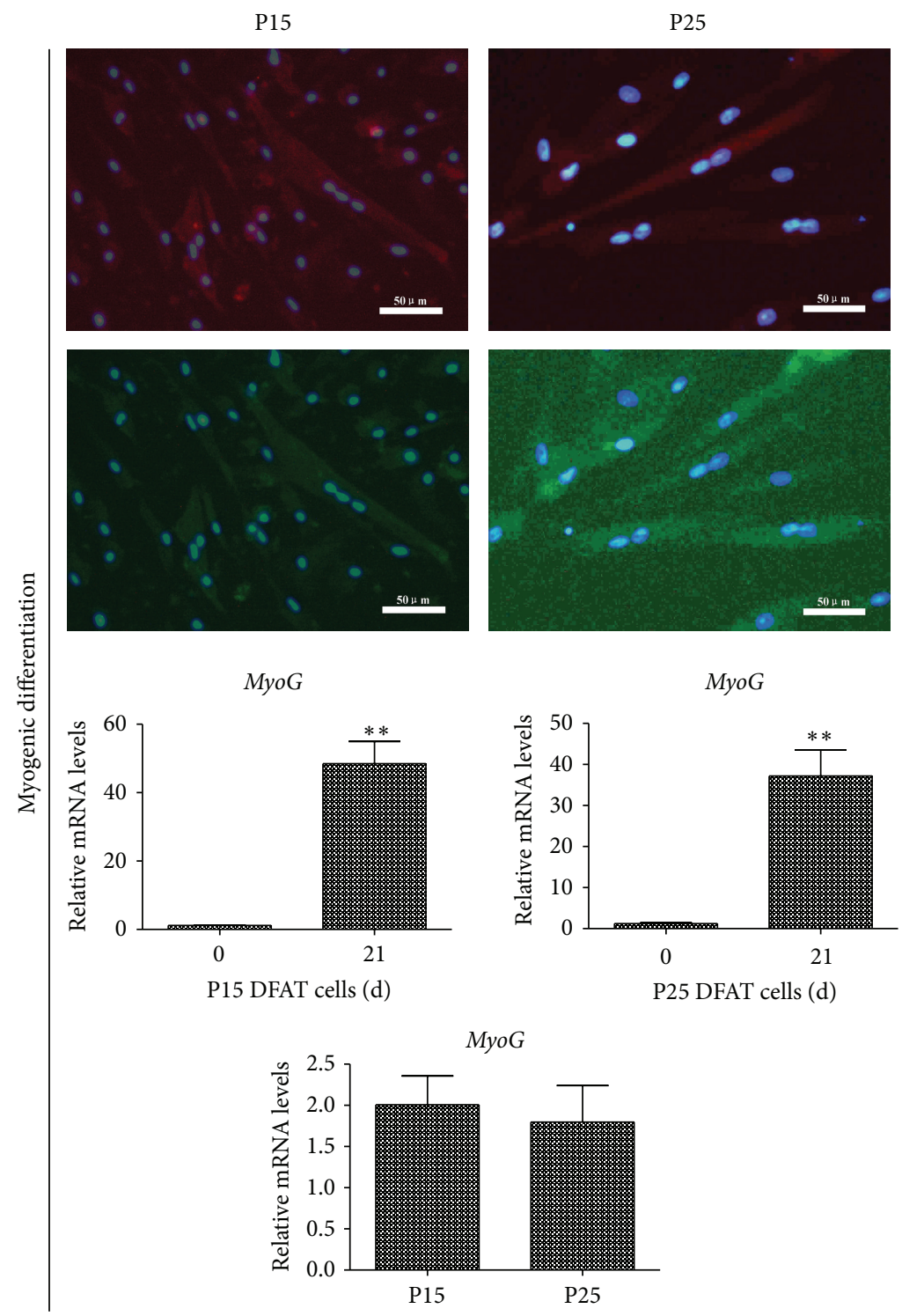

(c)

FIGURE 4: Multilineage differentiation of porcine DFAT cells. (a) Adipogenic differentiation of DFAT cells at P3, P20, and P50 was induced by MDI (high-glucose DMEM containing 10\% FBS, $1 \mu \mathrm{M}$ dexamethasone, $500 \mu \mathrm{M}$ isobutylmethylxanthine (IBMX), $10 \mu \mathrm{g} / \mathrm{mL}$ insulin, and $200 \mu \mathrm{M}$ indomethacin) for 12 days. Lipid droplets were stained by Oil Red O, bar, $50 \mu \mathrm{m}$. Expression of PPAR $\gamma$ mRNA was assessed by real-time PCR. (b) Osteogenic differentiation of DFAT cells at P3, P20, and P50 was induced by induction medium (high-glucose DMEM containing $10 \% \mathrm{FBS}, 0.1 \mu \mathrm{M}$ dexamethasone, $10 \mathrm{mM} \beta$-glycerophosphate, and $50 \mathrm{mM}$ ascorbic acid) for 21 days, bar, $200 \mu \mathrm{m}$. Expression of Runx 2 mRNA was assessed by real-time PCR. (c) Myogenic differentiation of DFAT cells at P15 and P25 was induced by Galectin-1 for 21 days, expression of myogenic markers was confirmed by immunofluorescence analysis for Desmin and MyHC, and the nuclei were stained by DAPI. Images were merged by Desmin (red) and MyHC (green) with DAPI (blue), bar, $50 \mu \mathrm{m}$. Expression of MyoG mRNA was assessed by real-time PCR. All values are represented as mean \pm SD from three independent experiments. ${ }^{*} p<0.05,{ }^{* *} p<0.01$.

similar to the continuous cell line, which is much smaller than human DFAT cells (30-70 h) and ASCs $(40-120 \mathrm{~h})[8,9]$. In addition, the CPD of porcine DFAT $(47.40 \pm 1.64$ in 58 days after 16 passages) is much higher than the one reported about human DFAT cells (14.5 \pm 7.3 in 88 days after 5 passages) [11]. These above mentioned results reveal that porcine DFAT exhibit potent proliferative potential even after long-term culture in vitro.
Previous studies found that DFAT cells that possess properties similar to MSCs have been multilineage potential, including adipocytes, osteoblasts, chondrocytes [9, 11, 27], myocytes [28], smooth muscle cells [29], cardiomyocytes [12], and vascular endothelial cells [30]. Moreover, due to the potent adipogenic differentiation capacity, porcine DFAT cells were used as preadipocytes models for adipogenic differentiation [26]. However, to our knowledge, apart from 
adipogenic differentiation, different lineages differentiation of DFAT cells has not been reported in pigs. Therefore, we evaluate adipogenic, osteogenic, and myogenic differentiation of porcine DFAT cells during long-term culture in vitro (Figure 4). The results indicate that porcine DFAT cells maintain ability to undergo differentiation of adipocytes, osteoblasts, and myocytes by prolonged passaging. In particular, the visible mineralized matrix aggregates and multinucleated myotubes were for the first time to confirm the osteogenic and myogenic differentiation of DFAT cells in pigs.

In summary, we success to establish the porcine DFAT cells by ceiling culture and provide evidence of morphology, cell viability, growth kinetics, differentiation, and karyotype, revealing that porcine DFAT cells exhibit efficient proliferative activity, multipotent differentiation to adipocytes, osteoblasts, and myocytes, normal diploid karyotype during the long-term culture in vitro. Because of the abundance and the easy accessibility of mature adipocytes, porcine DFAT cells can be an excellent stem cell model applied to tissue engineering and regenerative medicine.

\section{Conflict of Interests}

The authors declare that there is no conflict of interests regarding the publication of this paper.

\section{Acknowledgments}

This work is supported by the State Key Development Program for Basic Research of China (Grant no. 2013CB1273005), the Natural Science Foundation of Hubei province (Grant no. 2013CFA010), and the Fundamental Research Funds for the Central Universities (Grant no. 2013PY047).

\section{References}

[1] M. F. Pittenger, A. M. Mackay, S. C. Beck et al., "Multilineage potential of adult human mesenchymal stem cells," Science, vol. 284, no. 5411, pp. 143-147, 1999.

[2] P. A. Zuk, M. Zhu, H. Mizuno et al., "Multilineage cells from human adipose tissue: implications for cell-based therapies," Tissue Engineering, vol. 7, no. 2, pp. 211-228, 2001.

[3] M. V. Dodson, G. J. Hausman, L. Guan et al., "Skeletal muscle stem cells from animals I. basic cell biology," International Journal of Biological Sciences, vol. 6, no. 5, pp. 465-474, 2010.

[4] M. Shi, M. Ishikawa, N. Kamei et al., "Acceleration of skeletal muscle regeneration in a rat skeletal muscle injury model by local injection of human peripheral blood-derived CD133positive cells," Stem Cells, vol. 27, no. 4, pp. 949-960, 2009.

[5] D. Baksh, R. Yao, and R. S. Tuan, "Comparison of proliferative and multilineage differentiation potential of human mesenchymal stem cells derived from umbilical cord and bone marrow," Stem Cells, vol. 25, no. 6, pp. 1384-1392, 2007.

[6] C. Nombela-Arrieta, J. Ritz, and L. E. Silberstein, "The elusive nature and function of mesenchymal stem cells," Nature Reviews Molecular Cell Biology, vol. 12, no. 2, pp. 126-131, 2011.

[7] J. M. Gimble, A. J. Katz, and B. A. Bunnell, "Adipose-derived stem cells for regenerative medicine," Circulation Research, vol. 100, no. 9, pp. 1249-1260, 2007.
[8] H. Mizuno, M. Tobita, and A. C. Uysal, "Concise review: adipose-derived stem cells as a novel tool for future regenerative medicine," Stem Cells, vol. 30, no. 5, pp. 804-810, 2012.

[9] T. Matsumoto, K. Kano, D. Kondo et al., "Mature adipocytederived dedifferentiated fat cells exhibit multilineage potential," Journal of Cellular Physiology, vol. 215, no. 1, pp. 210-222, 2008.

[10] K. Yagi, D. Kondo, Y. Okazaki, and K. Kano, "A novel preadipocyte cell line established from mouse adult mature adipocytes," Biochemical and Biophysical Research Communications, vol. 321, no. 4, pp. 967-974, 2004.

[11] A. Poloni, G. Maurizi, P. Leoni et al., "Human dedifferentiated adipocytes show similar properties to bone marrow-derived mesenchymal stem cells," Stem Cells, vol. 30, no. 5, pp. 965-974, 2012.

[12] M. Jumabay, R. Zhang, Y. Yao, J. I. Goldhaber, and K. I. Boström, "Spontaneously beating cardiomyocytes derived from white mature adipocytes," Cardiovascular Research, vol. 85, no. 1, pp. $17-27,2010$

[13] M. E. Fernyhough, J. L. Vierck, G. J. Hausman, P. S. Mir, E. K. Okine, and M. V. Dodson, "Primary adipocyte culture: adipocyte purification methods may lead to a new understanding of adipose tissue growth and development," Cytotechnology, vol. 46, no. 2-3, pp. 163-172, 2004.

[14] A. S. de la Garza-Rodea, I. van der Velde-van Dijke, H. Boersma et al., "Myogenic properties of human mesenchymal stem cells derived from three different sources," Cell Transplantation, vol. 21, no. 1, pp. 153-173, 2012.

[15] R. Dariolli, V. Bassaneze, J. S. Nakamuta, S. V. Omae, L. C. G. Campos, and J. E. Krieger, "Porcine adipose tissue-derived mesenchymal stem cells retain their proliferative characteristics, senescence, karyotype and plasticity after long-term cryopreservation," PLoS ONE, vol. 8, no. 7, Article ID e67939, 2013.

[16] W. P. Cawthorn, E. L. Scheller, and O. A. MacDougald, "Adipose tissue stem cells meet preadipocyte commitment: going back to the future," Journal of Lipid Research, vol. 53, no. 2, pp. 227-246, 2012.

[17] B. Lindroos, R. Suuronen, and S. Miettinen, "The potential of adipose stem cells in regenerative medicine," Stem Cell Reviews and Reports, vol. 7, no. 2, pp. 269-291, 2011.

[18] J. Shen, A. Sugawara, J. Yamashita, H. Ogura, and S. Sato, "Dedifferentiated fat cells: an alternative source of adult multipotent cells from the adipose tissues," International Journal of Oral Science, vol. 3, no. 3, pp. 117-124, 2011.

[19] S. Wei, W. G. Bergen, G. J. Hausman, L. Zan, and M. V. Dodson, "Cell culture purity issues and DFAT cells," Biochemical and Biophysical Research Communications, vol. 433, no. 3, pp. 273275,2013

[20] Q. Gao, L. Zhao, Z. Song, and G. Yang, "Expression pattern of embryonic stem cell markers in DFAT cells and ADSCs," Molecular Biology Reports, vol. 39, no. 5, pp. 5791-5804, 2012.

[21] B. Gustafson and U. Smith, "Activation of canonical winglesstype MMTV integration site family (Wnt) signaling in mature adipocytes increases $\beta$-catenin levels and leads to cell dedifferentiation and insulin resistance," The Journal of Biological Chemistry, vol. 285, no. 18, pp. 14031-14041, 2010.

[22] H. Ono, Y. Oki, H. Bono, and K. Kano, "Gene expression profiling in multipotent DFAT cells derived from mature adipocytes," Biochemical and Biophysical Research Communications, vol. 407, no. 3, pp. 562-567, 2011.

[23] G. V. Røsland, A. Svendsen, A. Torsvik et al., "Long-term cultures of bone marrow-derived human mesenchymal stem cells 
frequently undergo spontaneous malignant transformation," Cancer Research, vol. 69, no. 13, pp. 5331-5339, 2009.

[24] H. Skottman and O. Hovatta, "Culture conditions for human embryonic stem cells," Reproduction, vol. 132, no. 5, pp. 691-698, 2006.

[25] L. E. Zaragosi, G. Ailhaud, and C. Dani, "Autocrine fibroblast growth factor 2 signaling is critical for self-renewal of human multipotent adipose-derived stem cells," Stem Cells, vol. 24, no. 11, pp. 2412-2419, 2006.

[26] H. Nobusue and K. Kano, "Establishment and characteristics of porcine preadipocyte cell lines derived from mature adipocytes," Journal of Cellular Biochemistry, vol. 109, no. 3, pp. 542-552, 2010.

[27] N. Kishimoto, Y. Momota, Y. Hashimoto, K. Ando, T. Omasa, and J. Kotani, "Dedifferentiated fat cells differentiate into osteoblasts in titanium fiber mesh," Cytotechnology, vol. 65, no. 1, pp. 15-22, 2013.

[28] T. Kazama, M. Fujie, T. Endo, and K. Kano, "Mature adipocytederived dedifferentiated fat cells can transdifferentiate into skeletal myocytes in vitro," Biochemical and Biophysical Research Communications, vol. 377, no. 3, pp. 780-785, 2008.

[29] T. Sakuma, T. Matsumoto, K. Kano et al., "Mature, adipocyte derived, dedifferentiated fat cells can differentiate into smooth muscle-like cells and contribute to bladder tissue regeneration," The Journal of Urology, vol. 182, no. 1, pp. 355-365, 2009.

[30] M. Jumabay, R. Abdmaulen, S. Urs et al., "Endothelial differentiation in multipotent cells derived from mouse and human white mature adipocytes," Journal of Molecular and Cellular Cardiology, vol. 53, pp. 790-800, 2012. 

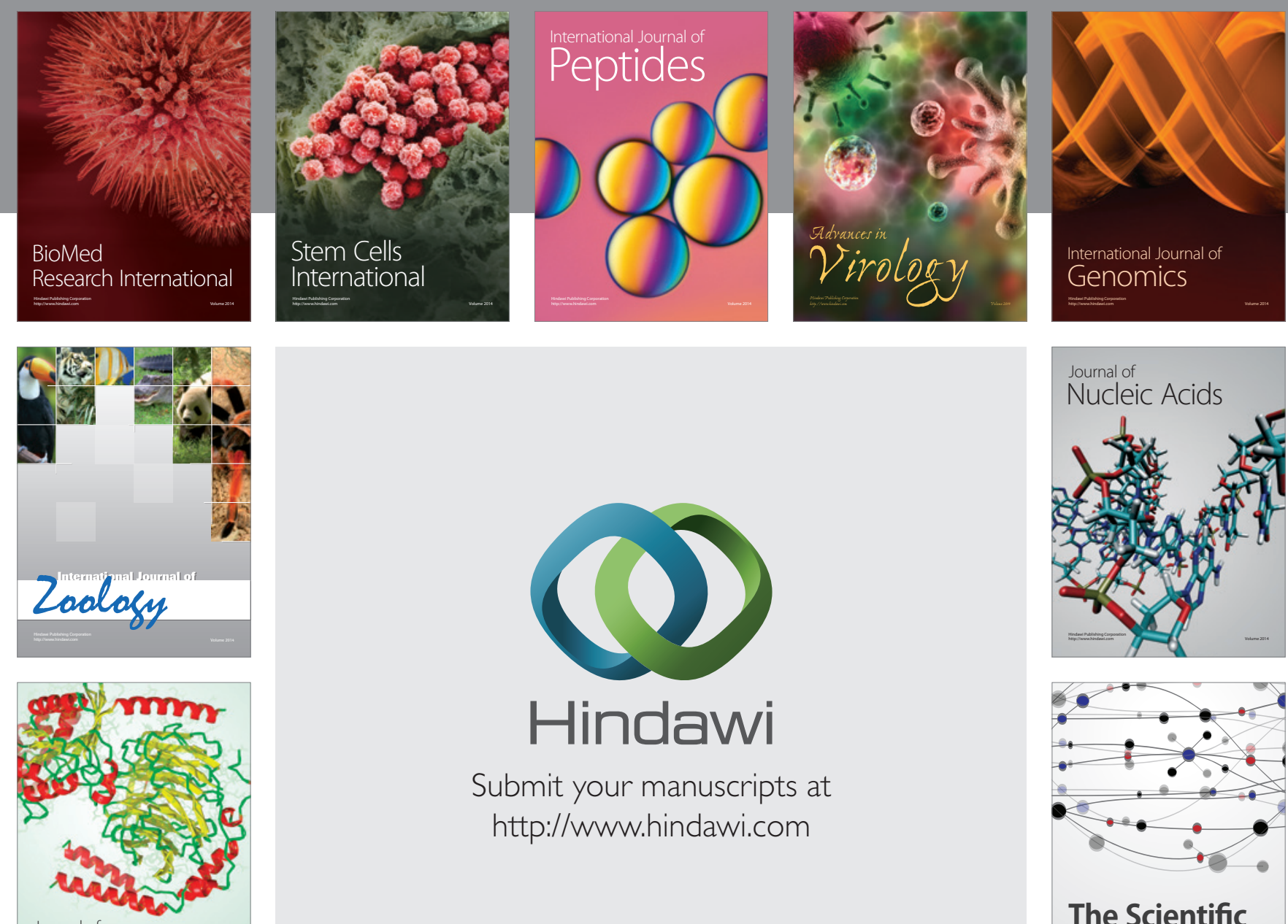

Submit your manuscripts at

http://www.hindawi.com

Journal of
Signal Transduction
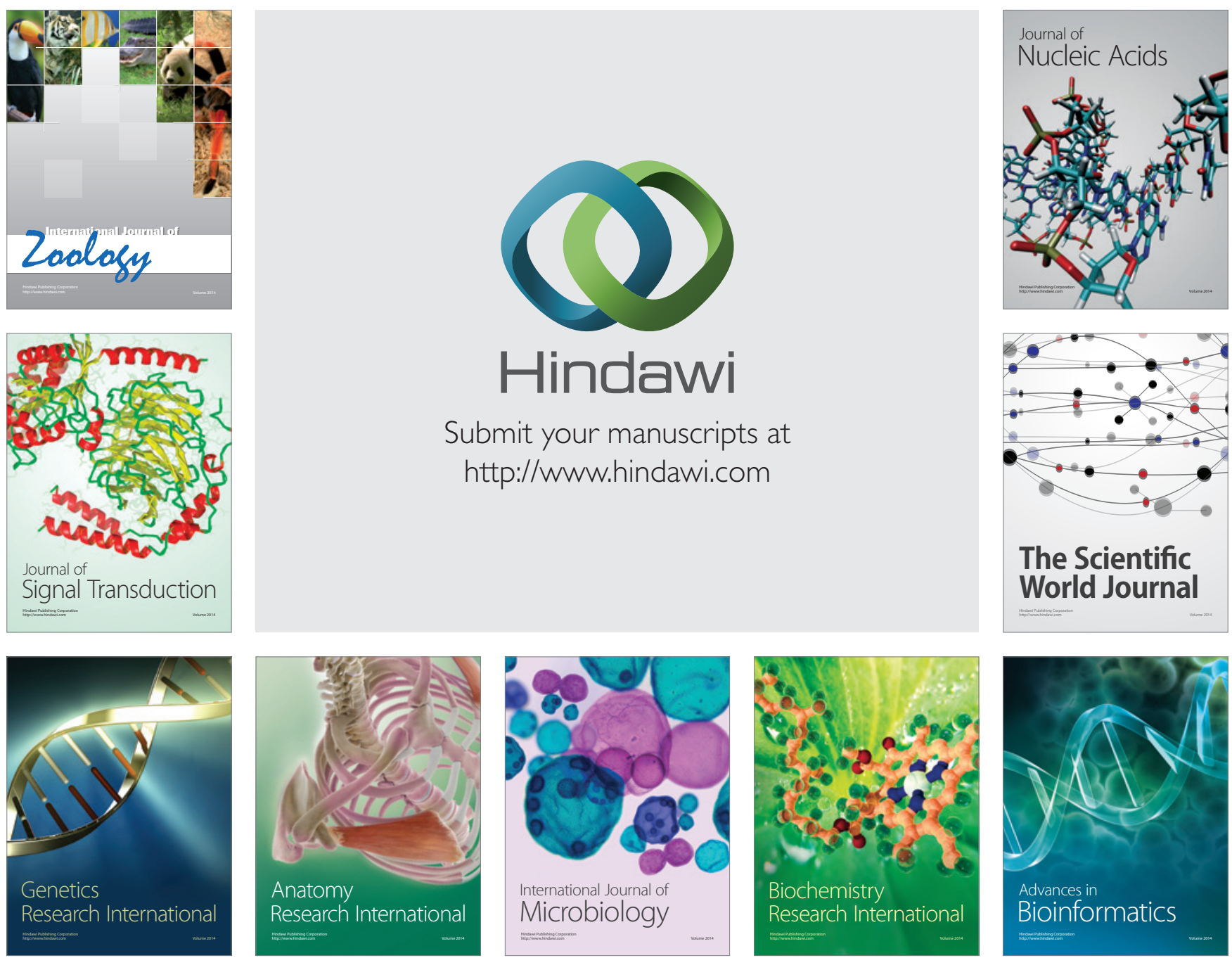

The Scientific World Journal
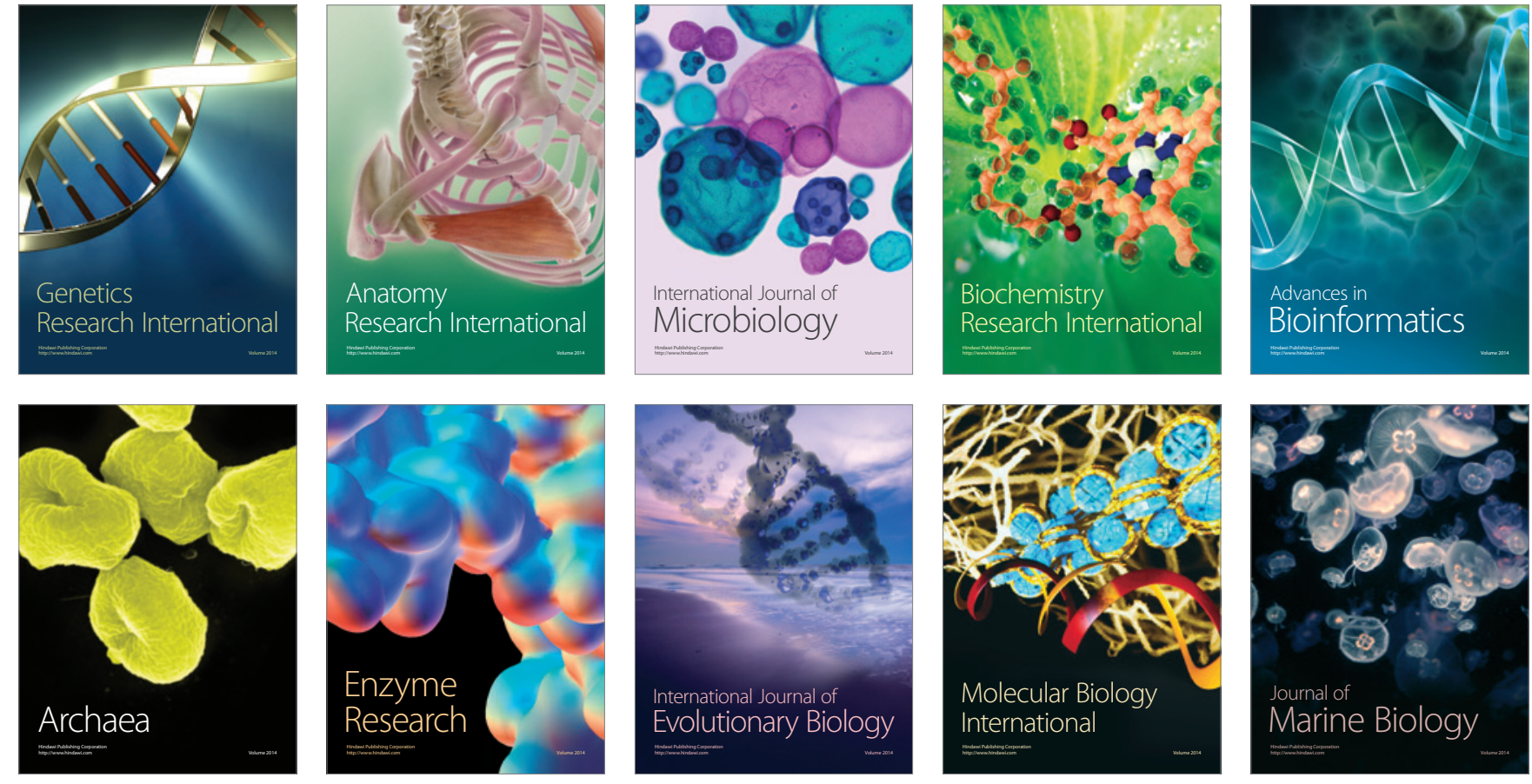